Cahiers « Mondes anciens »

ANCIENS

Histoire et anthropologie des mondes anciens

$11 \mid 2018$

La « civilisation » : critiques épistémologique et historique

\title{
L'invention de la tragédie selon Pierre Brumoy : de quelques pièges du relativisme
}

Brumoy and the invention of Tragedy: some issues of a relativist position

\section{Marie Saint Martin}

\section{(2) OpenEdition}

Journals

Édition électronique

URL : http://journals.openedition.org/mondesanciens/2141

DOI : $10.4000 /$ mondesanciens. 2141

ISSN : 2107-0199

Éditeur

UMR 8210 Anthropologie et Histoire des Mondes Antiques

Référence électronique

Marie Saint Martin, «L'invention de la tragédie selon Pierre Brumoy: de quelques pièges du

relativisme », Cahiers « Mondes anciens » [En ligne], 11 | 2018, mis en ligne le 13 mars 2018, consulté le 20 avril 2019. URL : http://journals.openedition.org/mondesanciens/2141 ; DOI : 10.4000/ mondesanciens. 2141

Ce document a été généré automatiquement le 20 avril 2019

\section{(i) $\$$}

Les Cahiers "Mondes Anciens " sont mis à disposition selon les termes de la licence Creative Commons Attribution - Pas d'Utilisation Commerciale - Pas de Modification 4.0 International. 


\title{
L'invention de la tragédie selon Pierre Brumoy : de quelques pièges du relativisme
}

\author{
Brumoy and the invention of Tragedy: some issues of a relativist position
}

\author{
Marie Saint Martin
}

1 Le $\mathrm{XVI}^{\mathrm{e}}$ siècle avait postulé une continuité et une forme d'universalisme unissant le goût antique au goût moderne pour légitimer son mouvement de retour à l'antique ; les débats qui s'ouvrent en France à partir de la querelle du Cid autour de la Poétique d'Aristote, au contraire, mettent en avant une rupture entre les deux périodes, rupture qui menaçait, si l'on suivait le clan des Modernes, de rendre illisibles et obsolètes les grands modèles grecs '. Dès ce moment, on le voit, les dés sont pipés, puisque c'est pour légitimer ou refuser les lectures aristotéliciennes du théâtre français que Chapelain, Corneille et les théoriciens qui leur emboîtent le pas invoquent les modèles d'Eschyle, de Sophocle ou d'Euripide, fourrant dans un même sac cet ensemble hétérogène que l'on appelle commodément les Anciens, et qui va d'Homère à Augustin. Se constitue alors un discours visant à réduire l'écart entre Eschyle et Aristote, et à faire de ce dernier le législateur d'une tragédie pourtant morte avant lui - Aristote et Eschyle se trouvant mis, en quelque sorte, sur un pied d'égalité et promus au rang d'inventeurs de la tragédie classique française.

2 Les réflexions de l'abbé Brumoy sur la naissance de la tragédie, inspirées de Plutarque ( Propos de table, I, 1) et d'Aristote (Poétique, chap. XI), opèrent, en 1730, une synthèse intéressante, parce qu'elle se veut globalisante, des différentes pistes avancées par le siècle précédant. Elles me paraissent exemplaires de la manière dont le classicisme français pense l'origine du théâtre et la notion d'invention, liées à ce qui n'est pas théorisé en termes de civilisation, mais de poli (ou de polissage) - une origine construite $a$ posteriori, destinée paradoxalement à justifier une linéarité qui conduit à un double geste : proclamer à la fois et dans le même temps la continuité de la Grèce classique à la France du XVII ${ }^{e}$ siècle, et la direction d'un progrès qui ne peut que sacrer les Français rois d'un genre inauguré par leurs illustres ancêtres, et porté par eux à son apogée. Cette double stratégie est destinée chez Brumoy à sortir de l'aporie installée par la querelle entre le 
parti des Anciens et celui des Modernes (mettant face à face une fidélité aux Anciens qui semble condamner les dramaturges à une fixité formelle et une modernité qui les soumet au relativisme du goût d'un public ignorant); elle s'appuie sur la théorisation d'un relativisme nuancé, qui permet d'échapper au double écueil que constituent, d'un côté, les critiques des Modernes à l'encontre de spectacles jugés trop barbares pour le public moderne, et de l'autre, la pratique philologique des Anciens, incapable selon Brumoy de rendre compte de l'esprit des originaux et de les faire apprécier. Comme il me semble que ce double principe demeure le postulat nécessaire à toute entreprise de traduction, voire même de lecture ou de mise en scène du théâtre antique, je voudrais tenter ici d'en mettre en évidence les apories autant que la fécondité. Je suivrai dans un premier temps la démarche archéologique de Brumoy afin de souligner que, contrairement aux apparences, la quête de l'origine est tournée vers l'avenir et non vers le passé, en ce qu'elle se définit par ce qui se trouve à la fin, c'est-à-dire par le théâtre classique, avant de montrer comment, à partir de cette démarche, Brumoy se trouve contraint d'affronter la question du relativisme, défini comme la prise de conscience de l'écart compromettant la possibilité d'une réception des textes antiques. Je conclurai par une série de questions visant à mesurer les conditions de possibilité du relativisme mesuré prôné par Brumoy, les problèmes qu'il résout et ceux qu'il ne manque pas de faire jaillir.

\section{La recherche paradoxale d'une origine grecque à la tragédie}

3 On assiste, chez Brumoy, à un discours de forme archéologique - classique depuis Aristote et la reconstitution qu'il propose de l'invention de la tragédie - dont l'objectif semble être de réécrire la Poétique, qui se voit ainsi justifiée et comme naturalisée :

Le besoin ou le plaisir ont porté les hommes à chercher les arts. Mais c'est au hasard et à la nature plutôt qu'à nos soins qu'ils doivent presque tous leur naissance. Les réflexions successives et réitérées ont ensuite perfectionné ce que la fortune avait comme offert d'elle-même; et ces réflexions en mûrissant, pour ainsi dire, et en se développant comme les germes de la nature, sont enfin passées en art; de sorte qu'on s'en est servi comme d'autant de principes établis, soit pour la mécanique, soit pour les lettres (Brumoy 1730, t. I, p. XXIX).

4 Si c'est par hasard (selon un modèle explicatif déjà développé chez Lucrèce) ou, mieux, par nature, que les arts se sont développés, le débat sur les règles, tel que la deuxième moitié du dix-septième siècle avait pu l'orchestrer, n'a plus lieu d'être. Corneille avait déjà avancé, dans le Premier discours sur le poème dramatique, l'idée que les règles trouvaient leur fondement dans le plaisir que pouvaient procurer les pièces qui les suivaient ${ }^{2}$; cependant, cet argument avait trouvé, chez Molière, dans La Critique de l'École des femmes, sa conséquence : si les règles destinées à fabriquer de bonnes pièces étaient responsables de pièces mauvaises, on pouvait - on devait - les changer ${ }^{3}$. En les naturalisant, Brumoy ôte aux règles d'Aristote leur statut de convention, et fonde l'art en nature, désamorçant de ce fait toute discussion sur leur légitimité. Or, l'argument qui permet cette manipulation est le constat qu'on «trouve de temps immémorial des traces d'œuvres Théâtrales en diverses nations polies ${ }^{4}$ qui ne s'étaient pas communiqué ce goût les unes aux autres (Brumoy 1730, p. XXX). " Se voient alors convoqués, grâce à la caution de différents savants, les exemples chinois et incas qui, au même titre que les Grecs et les Parisiens, peuvent prétendre au cercle des nations policées. La conclusion peut sembler circulaire : «tant il est vrai que les hommes se ressemblent partout, et que partout les 
arts d'imitation se puisent dans la même source, qui est la nature !» Un lecteur d'Aristote seul y retrouve ses petits : on n'y comprend rien si l'on ignore que l'imitation est l'une des opérations naturelles propres à l'homme, selon les premières pages de la Poétique (chap. IV). Les règles deviennent ainsi le développement naturel d'un art pratiqué par quelques peuples policés, et les remettre en question reviendrait à se ranger, soit du côté de barbares ignorants (ainsi, du théâtre baroque du début du XVII ${ }^{\mathrm{e}}$ siècle ou du théâtre anglais), soit du côté d'hommes dénaturés parce qu'ayant renié leur nature.

5 La stratégie argumentative de Brumoy résulte ensuite d'un double mouvement en apparence contradictoire, qui insiste sur la continuité en même temps qu'il met en valeur une série de ruptures. L'abbé commence par un récit qui, alors qu'il prétend rechercher l'origine du genre, fonctionne selon une logique générative ou cumulative bien plus qu'explicative : il rapporte «l'historiette » de Bacchus et du bouc à lui immolé, autour duquel dansent les paysans; "divertissement passager qui devint usage annuel, puis sacrifice public, ensuite cérémonie universelle, et enfin spectacle profane (Brumoy 1730, p.XXXII) ». S'ajoute une série d'éléments (musique, danse, dispute, etc.), passés par l'Égypte pour ce qui est des chœurs, et améliorés «par degrés ", à mesure que les Grecs venaient "à se polir ", si bien que les temples deviennent théâtres, selon une forme de transfiguration magique à laquelle on assiste d'une manière progressive, cette progressivité se muant en causalité sous la plume de Brumoy. D'une fête rurale en l'honneur d'un dieu, on est parvenu à de la poésie chantée, la seule permanence dans ce jeu d'anamorphoses étant le bouc: ce qui fonde la définition de la tragédie, son essence, c'est un nom, une étymologie plus ou moins fantaisiste mais héritée de la tradition. « On perfectionna de plus en plus le même genre; mais on ne le changea pas. " Cette dilution de la naissance de la tragédie dans une série de métamorphoses successives permet à Brumoy de repousser l'origine du genre dans un avant : en clair, la tragédie existait avant d'être née, en des temps immémoriaux, ce qui est normal puisqu'il s'agissait, on l'a vu, d'un processus naturel. Les explications de Brumoy, sous couvert de dévoiler l'origine de la tragédie, occultent donc le saut entre le moment où la tragédie n'est pas et celui où elle est, donnant ainsi l'illusion d'une continuité sur laquelle il insiste, puisqu'il évoque « plus de quinze ou seize poètes, presque tous successeurs les uns des autres ", qui finalement, par lassitude, se résignèrent à changer quelque chose au modèle initial pour le transformer en véritable tragédie. Ainsi, de tout temps, la tragédie a existé, et la tragédie classique française est le fruit de cet héritage linéaire. Or, la continuité remonte bien sûr à la seule origine possible en matière littéraire : Homère, qui de ce fait devient le véritable inventeur de la tragédie, puisque c'est chez lui que Thespis est allé chercher l'idée de montrer des "traits d'histoire ou de fable» (Brumoy 1730, p. XXXIV). La dilution de l'origine permet ainsi de revenir à l'origine incontournable de toute littérature occidentale : le vieil aède aveugle.

6 Mais Brumoy est aussi prisonnier d'un autre type de généalogie, celui qui procède, au contraire, par sauts et ruptures, d'un nom illustre à l'autre; malgré l'illusion de continuité construite autour du nom de tragoidia, la conclusion de l'abbé est claire :

On voit assez que ni dans ces hymnes, ni dans les Chœurs qui les chantaient, on ne trouve aucune trace de la véritable tragédie, à en pénétrer l'idée plutôt que le nom (p. XXXII).

7 La " véritable » tragédie, c'est sans doute la tragédie aristotélicienne sanctifiée par un siècle de pratique et de discussions françaises. Il faut donc trouver autre chose : Brumoy en vient à décrire une série d'innovations introduites par des inventeurs de génie ; sans 
surprise, on voit se dérouler la liste des grands noms, Thespis, Eschyle, Sophocle, etc. Mais quel que soit l'inventeur, on butte toujours sur le même problème, avoué au sujet de Thespis :

Mais après tout ces récits d'une action qu'on ne voyait pas, n'étaient qu'une espèce de Poème épique. En un mot il n'y a point encore là de vraie Tragédie (p. XXV).

Thespis est le premier, et en même temps il ne peut pas être le premier. On pourrait continuer : Eschyle sera le premier à introduire un dialogue, et en même temps il n'est pas le premier, car est-ce une véritable tragédie qu'un dialogue à deux personnages ? La succession de ruptures que permettent d'introduire les noms de Thespis, d'Eschyle ou de Sophocle, n'est qu'une manière de dissoudre ce qui demeure "l'embarras » irréductible de Brumoy - trouver le père de la tragédie, titre assigné de manière presque magique à Eschyle, dans une rupture qui pourrait sembler fondatrice, si elle n'était de nouveau nuancée :

Il est surprenant que ni Aristote, ni ceux qui ont traité cette matière, ne nous montrent pas avec précision les divers changements que reçut la Tragédie depuis sa naissance, jusqu'à sa maturité en Grèce. Il ne l'est pas moins qu'ils ne nous disent point nettement, excepté Philostrate et Quintilien, une chose qu'il faut toutefois nécessairement conclure de leurs écrits, à savoir, qu'Eschyle fut le véritable inventeur de la Tragédie proprement dite ${ }^{5}$. Tous en effet s'accordent à dire qu'il joignit un second Acteur à celui de Thespis. Voilà des interlocuteurs, voilà le Dialogue, et par conséquent un germe de la Tragédie. Avant lui rien de tout cela. C'est donc Eschyle qui en est le père (Brumoy 1730, p.XXXVI, à propos d'Aristote, Poétique, chap. 4).

9 Le problème demeure toutefois entier : si le nom d'Eschyle permet une nouvelle rupture, le choix de l'expression « un germe de la Tragédie » souligne qu'on n'est pas encore arrivé au bout du chemin... C'est que l'entreprise de Brumoy, celle de trouver l'inventeur de la "véritable tragédie ", est vouée à l'échec: sous le terme de "véritable tragédie », c'est semble-t-il la tragédie classique française qu'il faut entendre, et celle-ci est bien évidemment introuvable dans les modèles grecs. La tentative de Brumoy avance la restitution d'une origine, mais ne cesse de nous la dérober : c'est que l'origine n'est pas situable avant la tragédie française cornélienne - l'essence de la tragédie, c'est sa fin, ce qui pour Brumoy est sa définition inaltérable, la tragédie classique à la française. La stratégie de l'abbé est ainsi tournée vers le passé - puisqu'il s'agit de chercher la tragédie avant la tragédie, afin de mettre en évidence le lien direct qui unit, par nature, les peuples polis que sont les Grecs (comprenez: Homère, Eschyle, Sophocle, Euripide et Aristote) et les Français -, mais elle opère depuis un avenir qui est sa véritable origine. C'est ainsi que, par la transmission apparemment linéaire d'un genre tragique conçu comme une essence ayant de tout temps existé en puissance, avant même ses premières actualisations grecques, la nation française se trouve inscrite dans ce qui deviendra, quelques décennies plus tard, le grand récit de la civilisation occidentale, et qui n'est encore, sous la plume de Brumoy, que le grand récit des nations policées.

\section{Pour un relativisme nuancé}

De ce fait, Thespis, Eschyle ou Sophocle ne sont qu'autant d'étapes permettant de conduire en ligne directe d'Homère à Corneille : cela explique que Brumoy soit dans l'incapacité de fixer une borne finale à sa recherche (jamais on ne trouve, dans sa reconstitution, la « véritable tragédie », du moins chez les Grecs, et Brumoy se contente 
d'indiquer que «c'est dans ce point de maturité [une maturité encore jeune et verte] qu ['il va] désormais considérer l'art de la Tragédie ", "au point où les Grecs nous l'ont laissée »). On a vu que la source du genre se trouvait repoussée dans une nébuleuse qui remontait à Homère :

C'est dans ce point de maturité que je vais désormais considérer l'art de la Tragédie, pour en rechercher la vraie source dans l'esprit humain. C'est sans contredit Homère, je veux dire le Poème épique. [...] Le passage de l'épopée à la tragédie est plus naturel que celui des chœurs simples de Bacchus à l'invention de Thespis, si cependant cela même n'est pas dû à Homère (Brumoy 1730, p. XXXVII, à propos d'Aristote, chap. 4 et de Platon, Rép. 8).

11 Brumoy reconstitue alors, sous la forme d'une prosopopée pour le moins surprenante, les raisonnements qui ont dû nécessairement, et "naturellement", conduire Homère à inventer ce qui devait prendre la forme de l'Iliade et de l'Odyssée; or, ces raisonnements se trouvent énumérés comme une série de préceptes qui, eux, sont un copier-coller de la Poétique d'Aristote (règle du vraisemblable et du nécessaire, unité d'action, opposition entre le vrai historique et le vraisemblable de la fiction, etc.). L'Eschyle que fait parler Brumoy en fera de même quelques pages plus loin, justifiant les passions tragiques que sont la terreur et la pitié. Homère et Eschyle, concepteurs de génie, permettent ainsi à l'abbé de maintenir la rupture impliquée par la notion d'origine, alors même que cette dernière s'était trouvée diluée dans une succession d'inventions fondées par le hasard. L'origine de la prosopopée de ces deux auteurs est bien entendu la Poétique d'Aristote, telle que la conçoit le $\mathrm{XVII}^{\mathrm{e}}$ siècle - origine rétrospective, construite à partir des lectures que la modernité propose du texte antique. Brumoy est parfaitement conscient de ces difficultés, et il les expose d'une manière extrêmement honnête :

L'on me dira peut être qu'il n'est pas croyable que toutes ces réflexions aient passé par l'esprit d'Homère et d'Eschyle, quand ils se sont mis à composer, l'un son Iliade, et l'autre ses Tragédies; que ces idées paraissent postiches et venues après coup, qu'Aristote, charmé d'avoir démêlé dans leurs ouvrages de quoi fonder le but et l'art de l'épopée et de la Tragédie, a mis sur le compte de ces auteurs des choses auxquelles, selon les apparences, ils n'ont pas songé, etc. [...] Mais croira-t-on que ces grands hommes aient travaillé sans dessein ? [...] S'il est vrai qu'en effet l'art de la Tragédie résulte de leurs ouvrages, leur refusera-t-on le mérite de l'y avoir mis, et voudra-t-on leur ravir l'honneur d'avoir pu penser ce que nous n'avons pensé qu'après eux et par eux? (p. LIV).

C'est ici poser la question de l'auteur et de son intention: le geste de Brumoy aboutit, si on le pousse à son dernier terme, à faire d'Homère l'auteur de la Poétique d'Aristote, puisqu'il en applique si bien les préceptes.

Puisque les productions d'Eschyle obéissent à des règles naturelles, et que la Poétique est universalisable, il devient compliqué d'expliquer l'échec des modèles grecs sur la scène française ; c'est pourtant l'ambition que se fixe Brumoy. Pour cela, il récupère l'argument relativiste, mis au point par Corneille près d'un siècle plus tôt dans ses Trois Discours. Le problème de Brumoy comme de Corneille est qu'il faut parvenir à s'affranchir de deux publics un peu trop encombrants, et qui paralysent toute originalité: le public des Anciens, ces doctes frileux qui refusent toute concession dans la traduction des modèles grecs, au risque de les parer de couleurs ridicules qu'ils n'avaient pas dans l'original c'est du moins ce que Houdar de la Motte reproche à Anne Dacier, par exemple, lors de la querelle d'Homère; le public des Modernes, prêts à tout pour mettre les originaux au goût du jour, et qui n'hésitent pas, comme Quinault dans son Alceste, à défigurer Sophocle pour en faire une soupe conformiste et sans aucune originalité selon ses adversaires. 
Entre deux, reste à Corneille ou à Brumoy la possibilité de jouer avec les deux positions, en mâtinant l'argument relativiste cher aux Modernes d'une once d'universalisme prôné par les Anciens : c'est ce que permet le recours à la notion de progrès, appliquée à ce qui, dans le travail du dramaturge, est qualifié d'« art ». Dans une tragédie, se distinguent dès lors deux éléments: les "beautés ", universelles et audibles par tout homme quel qu'il soit ; l'art, qui est le fruit d'une méthode relative et en perpétuelle évolution. La notion de progrès, par sa linéarité, permet de sortir du temps cyclique dans lequel étaient enfermés les doctes, mais elle permet également de relier les deux sphères inconciliables qu'étaient l'Antiquité et le contemporain pour un certain nombre de Modernes; elle avait d'ailleurs déjà été mise en forme par l'un des représentants les plus célèbres du second parti, Perrault, pour instituer la tragédie lyrique comme fidèle continuatrice de la tragédie antique. Avant lui, Corneille estimait déjà, dans la préface de Clitandre, que :

Puisque les Sciences et les Arts ne sont jamais à leur période, il m'est permis de croire qu'ils [les Anciens] n'ont pas tout su, et que de leurs instructions on peut tirer des lumières qu'ils n'ont pas eues. Je leur porte du respect comme à des gens qui nous ont frayé le chemin, et qui après avoir défriché un pays fort rude nous ont laissé à le cultiver. J'honore les Modernes sans les envier, et n'attribuerai jamais au hasard ce qu'ils auront fait par science, ou par des règles particulières qu'ils se seront eux-mêmes prescrites (Corneille 1987, p. 95-96).

Le « respect » dû aux Anciens n'implique pas observation sans conditions de leurs règles, puisque les Modernes ont des « lumières » qu'ils n'avaient pas. L'Antiquité est comme une vieille femme qui fut belle, mais qu'on n'épouserait plus au XvII e siècle : «Ce n'est pas que je méprise l'antiquité, mais comme on épouse malaisément des beautés si vieilles, j'ai cru lui rendre assez de respect de lui partager mes ouvrages ", dit-il dans la préface de $L a$ Veuve (Corneille 1987, p. 203). Il ajoute, dans l'avertissement d'Agésilas :

Leurs règles sont bonnes, mais leur méthode n'est pas de notre siècle, et qui s'attacherait à ne marcher que sur leurs pas, ferait sans doute peu de progrès, et divertirait mal son auditoire (Corneille 1987, p. 563-564).

Le dédoublement que Corneille opère entre règles et méthode est déplacé par Brumoy, et devient une opposition entre la nature et l'éducation - il ressemble à ce que Florence Dupont dénonce lorsqu'elle parle d'une séparation arbitraire entre «ce qui relèverait de l'universel » et « ce qui serait de l'ordre de l'accident historique » (Dupont 2001, p. 14) - ; la prosopopée d'Homère s'emploie à désamorcer l'argument relativiste en soulignant cette difficulté :

Si l'on veut être goûté, on doit peindre les objets tels que la nature et l'éducation nous les offrent. Grand principe qui doit me justifier aux yeux de la postérité la plus reculée, si elle daigne se rappeler que les mœurs du siècle où j'écris auront été bien différentes des siennes (Brumoy 1730, p. XLIII).

L'argument repose sur une conception représentative de l'art poétique : puisque la poésie doit peindre le monde, il faut pardonner à la poésie antique de peindre un monde qui ne ressemble plus au nôtre. Les règles du théâtre classique sont immuables, et inchangées depuis Homère jusqu'au $\mathrm{xVIII}^{\mathrm{e}}$ siècle; il faut en revanche faire entrer en variance l'ensemble du contexte qui les entoure - comme si l'on pouvait séparer le fond de la forme, la fable de son actualisation. Ainsi, lorsque Brumoy condamne l'intrigue d'Électre comme trop «horrible», ce n'est pas la notion de vraisemblance qu'il est conduit à relativiser et à mettre en contexte, mais celle de la religion: le matricide, loin d'être compris comme un acte monstrueux et de ce fait spectaculaire, devient un exemple de la barbarie antique en matière de religion, et se trouve réduit au statut d'événement ordinaire aux yeux d'un Grec, renvoyant à une « sensibilité culturelle particulière », pour 
reprendre les mots de Florence Dupont au sujet du crime d'Oreste (2001, p. 29) : si, dans les paroles des Dioscures telles que les lit Brumoy, la justification de l'acte horrible repose sur la destinée, c'est la preuve non d'une modification de paradigme esthétique, mais d'une «morale étrange pour des Dieux!» (Brumoy 1730, p. 232-233). Brumoy, qui met l'accent sur le respect de la vraisemblance exigé par Aristote, comme le souligne Filippo Fassina (2011), en conclut que :

L'idée seule qu'on avoit alors de la fatalité, suffisoit pour diminuer l'horreur et

l'atrocité d'un parricide médité et commis de sang froid (Brumoy 1730, p. 197).

La religion, la politique, la morale - tout ce qui suffira au XIx siècle à définir une civilisation, et qui dessine, sous la plume de Brumoy, les bornes d'une « nation policée »sont relatives. Elles ne doivent cependant pas cacher la permanence des beautés tragiques, incarnées par les règles aristotéliciennes, immuables et universelles. L'ensemble de ces éléments constitue une sorte de langue étrangère, qui seule forme obstacle à la lecture; l'entreprise de Brumoy est donc une entreprise de traduction (linguistique et culturelle), qui doit permettre d'effacer les traces de ces mœurs relatives, pour reconstituer un théâtre grec lisible par ses contemporains - pour ce qu'il appelle luimême « rebâtir le théâtre ancien de ses propres débris ». Le théâtre grec ne peut ainsi être intégré au grand récit d'une continuité occidentale qu'au prix d'un important travail de lissage accompli par les Modernes. Entreprise vouée à une grande fortune, depuis la tragédie néoclassique $\mathrm{du} \mathrm{xVIII}^{\mathrm{e}}$ siècle jusqu'aux vastes péplums romantiques et aux projets de théâtre archéologique de la fin du XIX ${ }^{\mathrm{e}}$ siècle. Brumoy postule donc, sous cette langue que sont non seulement les mots grecs, mais aussi la religion, la politique, en somme la diversité des coutumes, une essence isolable, une « idée » de tragédie, capable si elle est bien traduite d'émouvoir de manière universelle - l'argument avait déjà été utilisé par Racine pour justifier son Iphigénie. La traduction devient un exercice de " dépaysement » (Brumoy 1730, p. XII), dans lequel le traducteur doit se montrer capable de détacher les beautés antiques $d u$ " défaut de leur siècle », d'adopter un " point de vue » qui est celui des auteurs, et de comprendre le « génie » des nations étudiées, afin de leur pardonner les nécessaires fautes de goût que leur immaturité explique, et de juger l'essentiel, ce qui se trouve sous la coutume et sa relativité :

Distinguons la vérité et la beauté d'avec les circonstances que l'éducation y ajoute : et de ces circonstances-là même tirons non seulement une raison plausible de tant de contradictions apparentes ou réelles dans le jugement qu'on porte des Anciens, mais encore une règle de précaution qu'on doit prendre dans la lecture de leurs ouvrages.

J'entends ici par vérité et beauté en fait de productions d'esprit, telles que sont les Tragédies, une imitation de la nature qui saisit l'âme, et qui fait dire, suivant les idées reçues dans une nation polie, cela est vrai, cela est beau. Je dis imitation de la nature suivant les idées reçues dans un pays où règne la politesse : car autant que la nature est uniforme dans ce qui appartient aux hommes en tant qu'hommes, dans le jeu des passions, par exemple; autant l'éducation varie-t-elle les intérêts qui meuvent les passions, et les manières de penser et d'agir (p. VIII-IX).

Tout se passe comme si, sous les traces variables et contingentes des coutumes grecques ou françaises, pouvait se découvrir la définition de ce qui réunit les différentes nations policées, les traits communs de ce que l'on pourrait appeler, par un léger anachronisme, la Civilisation ou, dans le vocabulaire de Brumoy, la politesse : le procédé permet, d'un peuple à l'autre, une compréhension fondée sur un héritage linéaire, malgré le nécessaire progrès qui rend l'un supérieur à l'autre - il permet, surtout, d'établir une continuité excluante d'Athènes à Paris, continuité dont se trouvent écartés, pour leur « rudesse », les 
Béotiens, équivalents sans doute des Anglais du $\mathrm{XVII}^{\mathrm{e}}$ siècle pour «l'air épais » qui règne chez eux et « passoit quelquefois jusqu'à l'esprit » (p. CXX).

On a beau sourire de cette entreprise, il me semble que tout projet de traduction ou d'adaptation du théâtre grec se heurte aux mêmes apories : postuler la possibilité d'une compréhension du théâtre grec, ou d'une traduction quelle qu'elle soit, c'est envisager derrière la diversité des mots ou des coutumes un contenu traductible, capable de toucher un spectateur du XVIII ${ }^{e}$ siècle tout autant qu'il touchait celui du v viècle av. J.-C., même s'il le fait d'une manière différente. De deux choses l'une: ou bien l'on récuse l'existence de ce contenu - ou, ce qui revient finalement au même, la possibilité d'y accéder; la traduction devient alors création, rêverie à partir des quelques traces qui nous restent de l'original, projection plutôt que réception, ce qui rend vain tout projet herméneutique; ou bien l'on conserve l'idée que l'on puisse accéder à quelque chose, qui n'est pas tout à fait l'original, de toutes façons dénué de sens hors contexte, mais qui n'est pas non plus uniquement le produit du cerveau enfiévré d'un moderne ; cette entreprise, nécessaire pour légitimer toute étude du contexte, qu'elle soit historique, philologique ou anthropologique, ne peut, me semble-t-il, se passer d'un minimum de commun entre l'Antiquité et nous - donc du postulat minimal de quelque chose d'universalisable. Or, et c'est peut-être ce que l'on peut tirer de la proposition de Brumoy, ce minimum universalisable pourrait bien se jouer dans les différentes transmissions qui nous font hériter de ce que nous appelons « les textes antiques » - l'Antiquité, ce serait alors non pas un objet appartenant au passé, mais la somme feuilletée, bien présente, de ces transmissions et héritages; si nous sommes touchés aujourd'hui encore par les pièces de Sophocle et d'Euripide, c'est sans doute aussi parce que l'histoire de ces transmissions, dont les traductions de Brumoy sont un jalon essentiel, les a transformées en classiques, même si c'est au prix d'une approche "texto-centrée", qui a fait de ces textes des tragédies françaises en cinq actes, les rendant ainsi lisibles aux lecteurs du $\mathrm{xvIII}^{\mathrm{e}}$ siècle et à ceux qui les suivirent.

20 Le père Brumoy, lorsqu'il met en forme l'argument relativiste comme instrument théorique destiné à répondre au parti des Modernes, en montrant la possibilité d'une lecture des Anciens débarrassée, grâce à un système de notes, des scories barbares qui émaillent leurs textes, invente une forme d'esthétique de la réception. On peut rapprocher ce projet de la démarche herméneutique dont Jauss lui-même (1967), lorsqu'il cherche à conjurer les apories de Schleiermacher (1837), ne parvient pas complétement à se défaire, en ce qu'elle consiste à reconstruire l'horizon d'attente des spectateurs pour lesquels le théâtre grec fut joué, en resituant la tragédie grecque dans ses déterminations premières - reconstitution qui a pour fin, chez Brumoy, contrairement à Jauss, de mieux l'en dégager. Cette pratique n'est pas exempte d'une série de pièges : elle conduit en premier lieu le père Brumoy à postuler, derrière le relativisme du goût, une forme de beau universel - postulat d'un commun nécessaire à Brumoy pour conserver la possibilité d'une lecture, voire d'une représentation, du théâtre des grecs comme de tout texte ancien. On peut se demander si cette construction d'un commun, et donc in fine ce retour à une forme d'universalisme, est évitable (voire, s'il est souhaitable de l'éviter), et quelles en sont les implications : faut-il cesser de lire le théâtre grec? peut-on le lire autrement que comme un particulier enfermé dans son temps auquel l'accès nous est définitivement fermé et accepter, comme Jauss finit par le faire du bout des lèvres après Gadamer, voire généraliser, le principe de la lecture anachronique? Si aucune interprétation herméneutique ne peut donner accès à l'événement dont ce texte est la trace, pas plus 
qu'aucune étude anthropologique d'ailleurs, faut-il pour autant abandonner l'effort de lecture et considérer le texte comme un énoncé mort? Le texte vit par ses lecteurs : c'est du moins le postulat de Brumoy lorsqu'il en propose une traduction destinée au plus grand nombre - le processus de vulgarisation, par le biais de la traduction et des notes, constituant une réponse en soi aux apories de l'herméneutique.

21 En second lieu, l'invention du relativisme va de pair avec la mise en parallèle de deux siècles, les anciens et les modernes, et avec la construction d'un "pays de l'Antiquité " (autrement appelé par Brumoy "siècle» ou "génie ancien»), au singulier, qui s'opposerait frontalement à l'ici et au maintenant des modernes, - deux cultures envisagées comme réconciliables par Brumoy, parce que pensables selon un paradigme commun, qu'on n'appelle pas encore civilisation, mais que l'on peut définir par ses acquis (la philosophie, la politique, la littérature). Entre un parti ancien qui, pour ses représentants les plus caricaturaux, transformait les textes grecs en poussière illisible et ridicule, et un parti moderne qui renonçait à les lire, et alors que tous deux postulaient l'altérité essentielle de l'Antiquité grecque par rapport à la Modernité, la solution élaborée par Brumoy pour continuer à lire ces textes est leur intégration à une culture commune, par la construction d'une communauté de "goût» - ce concept vide extrêmement volatile et construit ad hoc pour répondre aux interrogations du siècle ; on peut se demander si une autre solution est envisageable à qui souhaite traduire le théâtre grec. La réconciliation entre Anciens et Modernes opérée par Brumoy repose sur l'intégration d'Athènes au grand récit de la civilisation occidentale, et le refus de l'abandonner à une barbarie devenue illisible parce que ridicule. Cela pose la question encore une fois des études antiques : comment peut-on être antiquisant sans à son tour participer au grand récit de la civilisation occidentale, ne serait-ce qu'en héritant de cette étiquette, au singulier, "Antiquité " - et ce même alors qu'on s'attache, et qu'il faut continuer de s'attacher, à la décliner au pluriel, en toutes circonstances?

Enfin, la démarche intellectuelle de Brumoy conduit à sanctifier une conception référentielle de la littérature, pour laquelle restituer les conditions de production d'un théâtre doit permettre de mieux comprendre ce théâtre : c'est parce que les spectateurs de Sophocle vivent en "république » qu'ils peuvent prendre plaisir à voir sur scène le meurtre d'un roi, et c'est pour cette raison que les Modernes ne peuvent goûter ce genre d'action. L'échec du théâtre grec devant le public français serait imputable à la différence entre les cultures grecque et française : à l'imparfait polissage des Athéniens en matière politique ou morale répondent les imperfections de leurs tragédies. Dès lors, sauver les Grecs ne peut revenir qu'à traduire leurs productions, dans une tentative de reconstruction entièrement artificielle, et à transposer les réalités grecques dans le XVIII ${ }^{\mathrm{e}}$ siècle français, selon un projet assumé de « les faire parler français (autant qu'on le peut) comme ils parleraient eux-mêmes, s'ils faisaient passer leurs pensées en notre langue » (Brumoy 1730, p. XVIII). C'est poser le problème de tout transfert culturel : comment lire un texte irrémédiablement coupé de son contexte, sinon en le trahissant soit du côté d'un exotisme ridicule (ce que passent pour faire Dacier et le parti des Savants dans leurs traductions d'Homère notamment, dont le respect à l'égard du texte ne serait dès lors qu'un refus de traduire), soit du côté d'une déformation irrecevable du modèle? L'ambition de lire le théâtre grec autrement que comme objet archéologique définitivement illisible, coupé de son contexte de production et incapable de trouver un public, peut-elle alors être conservée ? À ces différentes questions, Brumoy me semble répondre avec un optimisme de bon aloi : oui, le théâtre grec reste vivant, puisqu'en tant 
que construction inévitablement rétrospective, il trouve son origine et sa raison d'être dans le lecteur contemporain qui en tire son inspiration et sa force innovante.

\section{BIBLIOGRAPHIE}

Brumoy P. (1730), Le Théâtre des Grecs, par le R. P. Brumoy, de la Compagnie de Jésus, Paris.

Corneille P. (1980, 1984, 1987), Euvres complètes, Couton G. éd., Paris.

Dacier A. (1692), La poétique d'Aristote, traduite en françois, avec des remarques, Paris.

Houdar de la Motte A. (1714), Discours sur Homère, Amsterdam.

Bastin-Hammou M. (2011), « Introduction. Brumoy, pédagogue et passeur du théâtre grec », dans Pascal J.-N. éd., Dossier " Le Théâtre des Grecs du Père Brumoy », Anabases 14, p. 27-41.

Dupont Fl. (2001), L'Insignifiance tragique : «Les Choéphores » d'Eschyle, «Électre » de Sophocle, « Électre » d'Euripide, Paris.

- (2007), Aristote ou le vampire du théâtre occidental, Paris.

Fassina F (2011), « Riccoboni e Brumoy. Due Dissertations sulla tragedia », dans Pascal J.-N. éd., dossier : «Le Théâtre des Grecs du Père Brumoy », Anabases 14, p. 101-112.

Gadamer H.-G. (1960), Wahrheit und Methode. Grundzüge einer philosophischen Hermeneutik, Tübingen.

Jauss H. R. (1967), Literaturgeschichte als Provokation der Literaturwissenschaft, Constance.

Lechevalier Cl. (2007), L'Invention d'une Origine. Traduire Eschyle en France de Lefranc de Pompignan à Mazon : Le Prométhée enchaîné, Paris.

Molière (1971), Euvres complètes, Couton G. éd., 2 vol., Paris.

Nietzsche F. (1872), Die Geburt der Tragödie aus dem Geiste der Musik, Leipzig.

Orfanos Ch. (2011), « La critique suspendue : le P. Brumoy et l'histoire », dans Pascal J.-N., dossier : "Le Théâtre des Grecs du Père Brumoy », Anabases 14, p. 43-59.

Rohde E. (1890-1894), Psyche. Seelenkult und Unsterblichkeitsglaube der Griechen, Leipzig.

Schleiermacher Fr. (1813), Über die verschiedenen Methoden des Übersetzens, Berlin.

- (1838), Hermeneutik und Kritik, Berlin.

Wilamowitz-Möllendorff U. (1872), Zukunftsphilologie : eine Erwidrung auf Friedrich Nietzsches "Geburt der Tragödie », Berlin.

- (2001), Qu'est-ce qu'une tragédie attique? Introduction à la tragédie grecque, Paris.

Zanin E. (2014), Fins tragiques: Poétique et éthique du dénouement dans la tragédie de la première modernité (Italie, France, Espagne, Allemagne), Paris. 


\section{NOTES}

1. Voir, en particulier, l'évolution des termes du débat entre la querelle du Cid et la querelle d'Alceste. C'est en fait avec les créations d'Horace et de Sophonisbe que Corneille affûte l'argument relativiste, pour le rendre plus efficace contre D'Aubignac et Donneau de Visé (voir en particulier l'« Avis au lecteur » de Sophonisbe). De cette querelle entre les Anciens et les Modernes, Molière donne un résumé, certes simplifié à l'extrême, dans le Malade imaginaire (Acte II, scène 6) : «Les Anciens sont les Anciens, et nous sommes les gens de maintenant. »

2. Corneille 1987, t. III, Discours de l'utilité et des parties du poème dramatique p. 117 : « Pour trouver ce plaisir qui lui est propre, et le donner aux spectateurs, il faut suivre les préceptes de l'art, et leur plaire selon ses règles. »

3. Uranie : «Pour moi, quand je vois une comédie, je regarde seulement si les choses me touchent ; et, lorsque je m'y suis bien divertie, je ne vais point demander si j'ai eu tort, et si les règles d'Aristote me défendaient de rire" (Molière 1971, t. I, Critique de l'École des femmes, Scène 6, p. 663).

4. Nous soulignons.

5. Nous soulignons.

\section{RÉSUMÉS}

Les réflexions de l'abbé Brumoy sur la naissance de la tragédie opèrent, en 1730, une synthèse des différentes pistes avancées par le siècle qui l'a précédé, et paraissent exemplaires de la manière dont le classicisme français pense l'origine du théâtre et la notion d'invention, le reliant à ce qui n'est pas théorisé en termes de civilisation, mais de politesse : une origine construite a posteriori, destinée paradoxalement à justifier une linéarité qui conduit à proclamer à la fois et dans le même temps la continuité de la Grèce classique à la France du XVII ${ }^{\mathrm{e}}$ siècle, et la direction d'un progrès qui ne peut que sacrer les Français rois d'un genre inauguré par leurs illustres ancêtres, et porté par eux à son apogée. Cette double stratégie, destinée chez Brumoy à réconcilier le parti des Anciens et celui des Modernes, s'appuie sur la théorisation d'un relativisme nuancé, qui permet d'échapper au double écueil que constituent, d'un côté, les critiques des Modernes à l'encontre de spectacles jugés trop barbares pour le public moderne, et de l'autre, la pratique philologique du parti des Anciens, incapable selon Brumoy de rendre compte de l'esprit des originaux et de les faire apprécier.

Brumoy's reflections on the birth of tragedy bring together, in 1730, the various propositions of the previous century; they seemingly epitomise the way French Classicism construes the origin of drama and the notion of invention, as it connects the former to what they theorize not as civilisation, but as politeness: an origin framed in retrospect, meant to justify paradoxically a linear conception which leads to claim both the continuity between classical Greece and 17thcentury France, and a line of progress by which the French nation is to be sacred as the leader of 
a genre that their famous ancestors have initiated, but that they have brought to its apogee. That two-fold strategy, which in Brumoy's mind ought to reconcile the Ancients and the Moderns, relies on the theorisation of a balanced relativism, thanks to which he avoids both the criticisms leveled by the Moderns at shows they judge too barbaric for a modern public, and the philological approach of the Ancients Brumoy thought was unable to translate the spirit of the originals and have them appreciated.

INDEX

Mots-clés : réception, querelle des Anciens et des Modernes, traduction, théâtre grec, naissance de la tragédie, Aristote, nations policées

Keywords : reception, quarrel of the Ancients and the Moderns, translation, greek theatre, birth of tragedy, Aristotle, polished nations

\section{AUTEUR}

\section{MARIE SAINT MARTIN}

Membre associée au CRLC (Paris-Sorbonne) et au CÉRÉdI (Université de Rouen) saintmartin.marie@orange.fr 